\title{
State Complexity of Neighbourhoods and Approximate Pattern Matching*
}

\author{
Timothy Ng, David Rappaport \\ Kai Salomaa \\ School of Computing, Queen's University \\ Kingston, Ontario K7L 3N6, Canada \\ tim.ng@uwaterloo.ca, \{daver, ksalomaa\}@cs.queensu.ca
}

\begin{abstract}
The neighbourhood of a language $L$ with respect to an additive distance consists of all strings that have distance at most the given radius from some string of $L$. We show that the worst case deterministic state complexity of a radius $r$ neighbourhood of a language recognized by an $n$ state nondeterministic finite automaton $A$ is $(r+2)^{n}-1$. In the case where $A$ is deterministic we get the same lower bound for the state complexity of the neighbourhood if we use an additive quasi-distance. The lower bound constructions use an alphabet of size linear in $n$. We show that the worst case state complexity of the set of strings that contain a substring within distance $r$ from a string recognized by $A$ is $(r+2)^{n-2}+1$. Keywords: regular languages, state complexity, lower bounds, additive distance
\end{abstract}

\section{Introduction}

The similarity of strings is often defined using the edit distance $[12,17]$, also known as the Levenshtein distance [15]. The edit distance is particularly useful for error-correction and error-detection applications $[1,8,9,10,11,13]$. A useful property is that the edit distance is additive with respect to concatenation of strings in the sense defined by Calude et al. [5].

If the distance of any two distinct strings of a language $L$ is greater than $r$, the language $L$ can detect up to $r$ errors [10, 12, 14] (assuming the errors have unit weight). Alternatively we can consider what the shortest distance is between strings in languages $L_{1}$ and $L_{2}$, that is, what is the smallest number errors that transform a string of $L_{1}$ into a string of $L_{2}$. Calude at al. [5] showed

*Electronic version of an article published as Int. J. Found. Comput. Sci. 29(2): 315329 (2018), DOI: doi.org/10.1142/S0129054118400099 copyright World Scientific Publishing Company www.worldscientific.com/worldscinet/ijfcs 
that the neighbourhood of a regular language with respect to an additive distance is always regular. Additive quasi-distances preserve regularity as well [5]. This gives rise to the question how large is the deterministic finite automaton (DFA) needed to recognize the neigbourhood of a regular language. Informally, determining the optimal size of the DFA for the neighbourhood gives the state complexity of error detection. Note that since complementation does not change the size of a DFA, the size of the minimal DFA for the neighbourhood of $L$ of radius $r$ equals to the state complexity of the set of strings that have distance at least $r+1$ from any string in $L$.

Schulz and Mihov [20] showed that a DFA recognizing the edit distance neighbourhood of fixed radius of a single word $w$ has at most $O(|w|)$ states. Povarov [18] showed that the Hamming neighbourhood of radius one of an $n$ state DFA language can be recognized by a DFA of size $n \cdot 2^{n-1}+1$ and also gave a lower bound $\frac{3}{8} n \cdot 2^{n}-2^{n-4}+n$ for its state complexity using languages defined over a binary alphabet. Using a weighted finite automaton construction the third author and Schofield [19] gave an upper bound of $(r+2)^{n}-1$ for the neighbourhood of radius $r$ of an $n$-state DFA language. No good lower bounds are known for neighbourhoods of radius at least two. Shamkin [22] has given a lower bound $2^{\left\lfloor\frac{n}{2}-r\right\rfloor}$ for the state complexity of radius $r$ Hamming neighbourhoods of an $n$ state finite language defined over a ternary alphabet. For a fixed number of states the lower bound is proportional to $2^{-r}$ and quite far from the known upper bound.

The string matching problem consists of finding occurrences of a particular string in a text [3]. El-Mabrouk [7] considers the problem of pattern matching with $r$ mismatches from a descriptional complexity point of view. Given a pattern $P$ of length $m$ and a text $T$, the problem is to determine whether $T$ contains substrings of length $m$ having characters differing from $P$ in at most $r$ positions, that is, substrings having Hamming distance at most $r$ from $P$. For a pattern $P=a^{m}$ consisting of occurrences of only one character, the state complexity was shown to be $\left(\begin{array}{c}m+1 \\ r+1\end{array}\right)[7]$.

The state complexity of $\Sigma^{*} L \Sigma^{*}$ was considered by Brzozowski, Jirásková, and $\mathrm{Li}$ [4] and was shown to have a tight bound of $2^{n-2}+1$. A DFA recognizing $\Sigma^{*} L \Sigma^{*}$ can be viewed to solve the exact string matching problem. In the terminology of Brzozowski et al. [4], $\Sigma^{*} L \Sigma^{*}$ is a two-sided ideal and the descriptional complexity of related subregular language families was studied recently by Bordihn et al. [2].

This paper studies the descriptional complexity of neighbourhoods and of approximate string matching. As our main result we give a lower bound $(r+$ $2)^{n}-1$ for the size of a DFA recognizing the radius $r$ neighbourhood of an $n$ state regular language. The lower bound matches the previously known upper bound [19]. The bound can be reached either using a neighbourhood of an $n$-state DFA language with respect to an additive quasi-distance or using a neighbourhood of an $n$ state NFA (nondeterministic finite automaton) language using an additive distance.

The lower bound constructions use an alphabet of size linear in $n$. A further limitation is that the (quasi-)distance associates different values to different 
edit operations. The precise state complexity of the edit distance with unit error costs remains open.

We also show that if $L$ is recognized by an $n$-state NFA the set of strings that contain a substring within distance $r$ from a string in $L$ with respect to an additive (quasi-)distance is recognized by a DFA of size $(r+2)^{n-2}+1$ and that this bound cannot be improved in the worst case. When $r$ is zero the result coincides with the state complexity of two-sided ideals [4].

\section{Preliminaries}

Here we briefly recall some definitions and notation used in the paper. For all unexplained notions on finite automata and regular languages the reader may consult the textbook by Shallit [21] or the survey by Yu [23]. A survey of distances is given by Deza and Deza [6] and the notion of quasi-distance is from Calude et al. [5].

We denote by $\Sigma$ a finite alphabet, $\Sigma^{*}$ the set of words over $\Sigma$, and $\varepsilon$ the empty word. A nondeterministic finite automaton (NFA) is a tuple $A=\left(Q, \Sigma, \delta, q_{0}, F\right)$ where $Q$ is a finite set of states, $\Sigma$ is an alphabet, $\delta$ is a multi-valued transition function $\delta: Q \times \Sigma \rightarrow 2^{Q}, q_{0} \in Q$ is the initial state, and $F \subseteq Q$ is a set of final states. We extend the transition function $\delta$ to $Q \times \Sigma^{*} \rightarrow 2^{Q}$ in the usual way. A word $w \in \Sigma^{*}$ is accepted by $A$ if $\delta\left(q_{0}, w\right) \cap F \neq \emptyset$ and the language recognized by $A$ consists of all strings accepted by $A$. The automaton $A$ is a deterministic finite automaton (DFA) if, for all $q \in Q$ and $a \in \Sigma, \delta(q, a)$ either consists of one state or is undefined. The definition allows the possibility of undefined transitions and, unless otherwise mentioned, we allow a DFA to be incomplete. A DFA $A$ is said to be complete if $\delta(q, a)$ is defined for all $q \in Q$ and $a \in \Sigma$.

The minimal DFA for a regular language $L$ is unique. For a given regular language $L$, the number of states of the minimal incomplete and minimal complete DFA recognizing $L$ differ by at most one. The state complexity of $L$, denoted by $\operatorname{sc}(L)$, is the size of the minimal DFA recognizing $L$. Note that we measure state complexity using incomplete DFAs.

Two states $p$ and $q$ of a DFA $A$ are equivalent (or indistinguishable) if $\delta(p, w) \in F$ if and only if $\delta(q, w) \in F$ for every string $w \in \Sigma^{*}$. A DFA $A$ is minimal if each state $q \in Q$ is reachable from the initial state and no two states are equivalent. The (right) Kleene congruence of a language $L \subseteq \Sigma^{*}$ is the relation $\equiv_{L} \subseteq \Sigma^{*} \times \Sigma^{*}$ defined by setting

$$
x \equiv_{L} y \text { iff }\left[\left(\forall z \in \Sigma^{*}\right) x z \in L \Leftrightarrow y z \in L\right] .
$$

The language $L$ is regular if and only if the index of $\equiv_{L}$ is finite and, in this case, the index of $\equiv_{L}$ is equal to the size of the minimal complete DFA for $L$ [21]. 


\subsection{Neighbourhoods of regular languages}

A function $d: \Sigma^{*} \times \Sigma^{*} \rightarrow[0, \infty)$ is a distance if it satisfies for all $x, y, z \in \Sigma^{*}$ the conditions $d(x, y)=0$ if and only if $x=y, d(x, y)=d(y, x)$, and $d(x, z) \leq$ $d(x, y)+d(y, z)$. The function $d$ is a quasi-distance [5] if it satisfies conditions 2 and 3 and $d(x, y)=0$ if $x=y$; that is, a quasi-distance between two distinct elements can be zero. In the following, unless otherwise mentioned, we consider only integral (quasi-)distances; that is, $d$ is always a function $\Sigma^{*} \times \Sigma^{*} \rightarrow \mathbb{N}_{0}$.

The neighbourhood of a language $L$ of radius $r$ is the set

$$
E(L, d, r)=\left\{x \in \Sigma^{*} \mid(\exists w \in L) d(x, w) \leq r\right\} .
$$

A distance $d$ is finite if for all nonnegative integers $r$ the neighbourhood of radius $r$ of any string with respect to $d$ is finite. A distance $d$ is additive [5] if for every factorization of a string $w=w_{1} w_{2}$ and radius $r \geq 0$,

$$
E(w, d, r)=\bigcup_{r_{1}+r_{2}=r} E\left(w_{1}, d, r_{1}\right) \cdot E\left(w_{2}, d, r_{2}\right) .
$$

A neighbourhood of a regular language with respect to an additive quasidistance is regular [5].

We will use the NFA construction for a neighbourhood of a regular language due to Povarov [18]. Informally, the construction makes $r+1$ copies of an NFA $A$, with each copy corresponding to a cumulative error ranging from 0 to $r$. A transition from a level $i$ to a level $i^{\prime}>i$ occurs when there is a transition that does not exist in $A$. There are $r+1$ such copies of $A$ to allow for at most $r$ errors. Strictly speaking, [18] deals with the Hamming distance but exactly the same construction works for any additive quasi-distance. We state the upper bound of [18] for quasi-distances and briefly recall also the proof because it will be used later in our lower bounds constructions.

Proposition 1 ([18]) If $A$ is an NFA with $n$ states and $d$ is an additive quasidistance, then $E(L(A), d, r)$ has an $N F A$ of size $n \cdot(r+1)$.

Proof. Consider an NFA $A=\left(Q, \Sigma, \delta, q_{0}, F_{A}\right)$. The neighbourhood $E(L(A), d, r)$ is recognized by an NFA

$$
B=\left(Q \times\{0,1, \ldots, r\}, \Sigma, \gamma,\left(q_{0}, 0\right), F_{A} \times\{0,1, \ldots, r\}\right),
$$

where the transitions of $\gamma$ are defined by setting for $q \in Q, 0 \leq i \leq r$ and $b \in \Sigma$ :

$$
\gamma((q, i), b)=\{(p, i+d(b, c)) \mid c \in \Sigma, p \in \delta(q, c), i+d(b, c) \leq r\} .
$$

The first component of the state of $B$ simulates a computation of $A$ on some string that possibly differs from the actual input string, and the second component keeps track of the "cumulative error". The computation accepts when the first component reaches a final state of $A$ and the cumulative error is at most $r$. 
To conclude this section, we derive an upper bound for the deterministic state complexity of the neighbourhood of a regular language with respect to an additive quasi-distance. Similar bounds are implied by the weighted finite automaton constructions in [16, 19], but the construction given in [19] deals only with neighbourhoods of a regular language with respect to a distance (as opposed to a quasi-distance). For completeness we give here a direct construction based on the NFA construction of Proposition 1. Before stating the bound in Proposition 2 we need the following lemma.

Lemma 1 Let $d$ be a quasi-distance, $r \in \mathbb{N}$ and let $A$ be an NFA with set of states $\{1, \ldots, n\}$. Let $D$ be the NFA with set of states $\{1, \ldots, n\} \times\{0, \ldots, r\}$ given by Proposition 1. Let $C$ be the DFA constructed from $D$ using the standard subset construction. If $(i, j)$ and $(i, j+k),(1 \leq i \leq n, 0 \leq j$ and $j+k \leq r)$ belong to a set $X$ with $k \geq 1$, then the sets $X$ and $X \backslash\{(i, j+k)\}$ are indistinguishable as states of $C$.

Proof. Consider a word $w=u v$ that reaches both $(i, j)$ and $(i, j+k)$ on the prefix $u$. If there is a computation on the suffix $v$ that takes the machine from $(i, j+k)$ to an accepting state $(f, l)$, then there also exists an accepting computation from $(i, j)$ to an accepting state $(f, l-k)$. Thus, any string that in $C$ has an accepting computation originating from $X$ also has an accepting computation originating from $X \backslash\{(i, j+k)\}$. The converse naturally holds since $X \backslash\{(i, j+k)\} \subseteq X$.

For the next construction recall that we consider only integral (quasi-)distances.

Proposition 2 For an n-state NFA $A$ and an additive quasi-distance $d$, the neighbourhood $E(L(A), d, r), r \in \mathbb{N}$ can be recognized by a DFA with $(r+2)^{n}-1$ states.

Proof. Let the set of states of $A$ be $\{1, \ldots, n\}$. Let $D$ be the NFA with set of states $\{1, \ldots, n\} \times\{0, \ldots, r\}$ recognizing $E(L(A), d, r)$ constructed as in the proof of Proposition 1 (due to [18]) and let $C$ be the DFA obtained from $D$ using the subset construction. By Lemma 1 , we can identify equivalent states in $C$ to obtain a DFA $C^{\prime}$ where all states are of the form $\left\{\left(i_{1}, j_{1}\right), \ldots,\left(i_{z}, j_{z}\right)\right\}$ where $1 \leq i_{1}<i_{2} \leq \cdots<i_{z} \leq n$ and $j_{1}, \ldots, j_{z} \in\{0, \ldots, r\}$, that is, the state sets of $C^{\prime}$ need to keep, for each $1 \leq i \leq n$, only the least value $j \in\{0, \ldots, r\}$ such that $(i, j)$ is in the corresponding set of states of $C$. Thus, in a state of $C^{\prime}$, for each $1 \leq$ $i \leq n$, we can encode possibilities for the second component as $0,1, \ldots, r, r+1-$ the value $r+1$ corresponding to the case that $i$ does not belong to the set as a first component. Furthermore, the set $\{(1, r+1),(2, r+1), \ldots,(n, r+1)\}$ is the dead state and can be omitted from $C^{\prime}$.

\section{State Complexity of Additive Neighbourhoods}

As the main result of this section we give a tight lower bound for the state complexity of a neighbourhood of a regular language given by a DFA (respectively, 
by an NFA) with respect to an additive quasi-distance (respectively, an additive distance).

For $n \in \mathbb{N}$ we consider an alphabet

$$
\Sigma_{n}=\left\{a_{1}, \ldots, a_{n-1}, b_{1}, \ldots, b_{n}, c_{1}, \ldots, c_{n-1}\right\} .
$$

For $r \in \mathbb{N}$, we define a quasi-distance $d_{r}: \Sigma_{n}^{*} \times \Sigma_{n}^{*} \rightarrow \mathbb{N}_{0}$ by the conditions:

- $d_{r}\left(a_{i}, a_{j}\right)=r+1$ for $i \neq j$,

- $d_{r}\left(b_{i}, b_{j}\right)=1$ for $i \neq j$,

- $d_{r}\left(a_{i}, b_{j}\right)=d_{r}\left(c_{i}, b_{j}\right)=r+1$ for all $1 \leq i \leq n-1,1 \leq j \leq n$,

- $d_{r}\left(a_{i}, c_{i}\right)=0$ for $1 \leq i \leq n-1$,

- $d_{r}\left(c_{i}, c_{j}\right)=r+1$ for all $1 \leq i, j \leq n-1$,

- $d_{r}\left(a_{i}, c_{j}\right)=r+1$ for all $i \neq j$,

- $d_{r}(\sigma, \varepsilon)=r+1$ for all $\sigma \in \Sigma$.

Note that the value $d_{r}(\sigma, \varepsilon)$ denotes the cost of the deletion and insertion operations and that the listed substitution, insertion, and deletion operations on elements of $\Sigma_{n}$ define a unique additive quasi-distance of $\Sigma_{n}^{*}[5]$.

Lemma 2 The function $d_{r}$ is an additive quasi-distance.

Proof. Clearly the function $d_{r}$ satisfies the triangle-inequality. We verify that $d_{r}$ is additive.

Let $r_{0} \geq 0$ and consider the neighbourhood $E\left(w_{1} w_{2}, d_{r}, r_{0}\right)$ of a word $w=$ $w_{1} w_{2}$. If $x \in E\left(w, d_{r}, r_{0}\right)$, then $d_{r}(x, w) \leq r_{0}$. We can consider the substitution of some symbol with another symbol or the empty string $\varepsilon$ as an edit operation with the cost defined by $d_{r}$. We may perform edit operations in any order. Thus, we can factor $x$ into two parts $x_{1} x_{2}$ in such a way that we obtain $w_{1}$ from $x_{1}$ with the cost of the operations summing to $d\left(w_{1}, x_{1}\right)$ and do the same to obtain $w_{2}$ from $x_{2}$ with cost $d\left(w_{2}, x_{2}\right)$. We then have $d_{r}\left(x_{1}, w_{1}\right)+d_{r}\left(x_{2}, w_{2}\right)=$ $d_{r}(x, w) \leq r_{0}$ and thus,

$$
x \in \bigcup_{r_{1}+r_{2}=r_{0}} E\left(w_{1}, d_{r}, r_{1}\right) \cdot E\left(w_{2}, d_{r}, r_{2}\right) .
$$

Conversely, if we have $x_{1} \in E\left(w_{1}, d_{r}, r_{1}\right)$ and $x_{2} \in E\left(w_{2}, d_{r}, r_{2}\right)$ with $r_{1}+r_{2}=$ $r_{0}$, then $d_{r}\left(x_{1}, w_{1}\right)+d_{r}\left(x_{2}, w_{2}\right) \leq d_{r}(x, w)$ and $x \in E\left(w, d_{r}, r_{0}\right)$.

We define the following family of incomplete DFAs. Let $A_{n}=\left(Q_{n}, \Sigma_{n}, \delta, 1,\{n\}\right)$ be a DFA with $n$ states where $Q_{n}=\{1, \ldots, n\}$ and $\Sigma_{n}$ is as in (1). The transition function $\delta$ is defined by setting

- $\delta\left(i, a_{i}\right)=i+1$ for $1 \leq i \leq n-1$

- $\delta\left(i, a_{j}\right)=i$ for $1 \leq i \leq n-2$ and $i+1 \leq j \leq n-1$ 


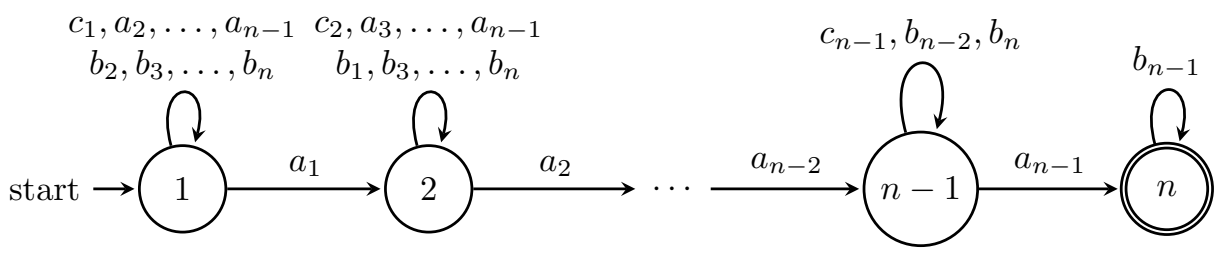

Figure 1: The DFA $A_{n}$

- $\delta\left(i, b_{j}\right)=i$ for $1 \leq i \leq n-1$ and $j=i-1$ or $i+1 \leq j \leq n$

- $\delta\left(i, c_{i}\right)=i$ for $1 \leq i \leq n-1$

All transitions not listed are undefined. The DFA $A_{n}$ is depicted in Figure 1.

The quasi-distance $d_{r}$ identifies the symbols $a_{i}$ and $c_{i}, 1 \leq i \leq n-1$. Using two different symbols that have distance zero in our quasi-distance allows us to define $A_{n}$ to be deterministic. By identifying $a_{i}$ and $c_{i}$ we can later modify the construction to give a lower bound for the neighbourhood of a language recognized by an NFA with respect to a distance (see Lemma 5).

To establish a lower bound for the state complexity of the neighbourhood $E\left(L\left(A_{n}\right), d_{r}, r\right)$ we define a set $S$ of strings that are all pairwise inequivalent with respect to the Kleene congruence of the neighbourhood. First we construct an NFA $B_{n, r}$ for $E\left(L\left(A_{n}\right), d_{r}, r\right)$ and the inequivalence of the strings in $S$ is verified using properties of $B_{n, r}$.

Suppose we have a DFA $A=\left(Q, \Sigma, \delta, q_{0}, F\right)$. Using Proposition 1 (due to [18]), an NFA $B=\left(Q^{\prime}, \Sigma, \delta^{\prime}, q_{0}^{\prime}, F^{\prime}\right)$ which recognizes the neighbourhood of radius $r$ of $L(A)$ with respect to a quasi-distance $d$ is defined by setting $Q^{\prime}=Q \times\{0, \ldots, r\}, q_{0}^{\prime}=\left(q_{0}, 0\right), F^{\prime}=F \times\{0, \ldots, r\}$ and the transitions of $\delta^{\prime}$ for $q \in Q, 0 \leq k \leq r$ and $a \in \Sigma$ are defined as

$$
\delta^{\prime}((q, k), a)=(\delta(q, a), k) \cup \bigcup_{b \in(\Sigma \cup\{\varepsilon\}) \backslash\{a\}}\{(\delta(q, b), k+d(a, b)) \mid k+d(a, b) \leq r\} .
$$

Now as described above we construct the NFA

$$
B_{n, r}=\left(Q_{n}^{\prime}, \Sigma_{n}, \delta^{\prime}, q_{0}^{\prime}, F^{\prime}\right),
$$

shown in Figure 2, which recognizes the neighbourhood of $L\left(A_{n}\right)$ of radius $r$ with respect to the quasi-distance $d_{r}$, where $Q_{n}^{\prime}=Q_{n} \times\{0,1, \ldots, r\}, q_{0}^{\prime}=(1,0)$, $F^{\prime}=F \times\{0,1, \ldots, r\}$ and the transition function $\delta^{\prime}$ is defined by

- $\delta^{\prime}\left((q, j), a_{q}\right)=\{(q, j),(q+1, j)\}$ for $1 \leq q \leq n-1$,

- $\delta^{\prime}\left((q, j), a_{q^{\prime}}\right)=\{(q, j)\}$ for all $1 \leq q \leq n-1$ and $q \leq q^{\prime} \leq n-1$,

- $\delta^{\prime}\left((q, j), b_{i}\right)=\{(q, j+1)\}$ for $1 \leq q \leq n$ and $i=1, \ldots, q-2, q$,

- $\delta^{\prime}\left((q, j), b_{i}\right)=\{(q, j)\}$ for $1 \leq q \leq n$ and $i=q-1, q+1, \ldots, n$, 


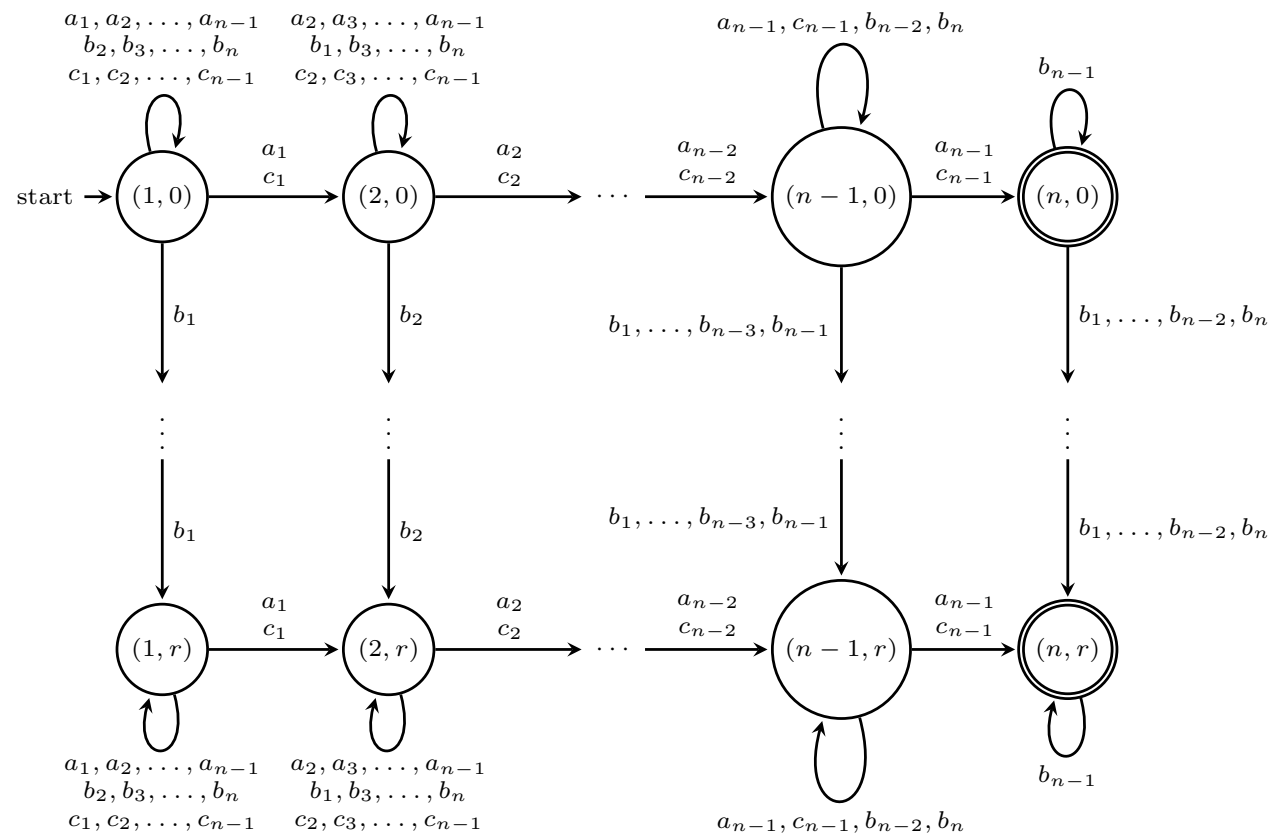

Figure 2: The NFA $B_{n, r}$

- $\delta^{\prime}\left((q, j), c_{q}\right)=\{(q, j),(q+1, j)\}$ for $1 \leq q \leq n-1$.

All transitions not listed above are undefined. Note that since in the distance $d_{r}$ the cost of inserting/deleting a symbol is $r+1$ and $B_{n, r}$ recognizes a neighbourhood of radius $r$ there are no error transitions (which are depicted as vertical transitions in Figure 2) corresponding to insertion/deletion. For the same reason the only error transitions for substitution correspond to substituting $b_{i}$ with $b_{j}, i \neq j$. The distance between $a_{i}$ and $c_{i}$ is zero (no error), and all other substitutions have cost $r+1$.

For $0 \leq k_{i} \leq r+1,1 \leq i \leq n$, we define the string

$$
w\left(k_{1}, \ldots, k_{n}\right)=a_{1} b_{1}^{k_{1}} a_{2} b_{2}^{k_{2}} \cdots a_{n-1} b_{n-1}^{k_{n-1}} b_{n}^{k_{n}} .
$$

The next lemma establishes a technical property of the computations of the NFA $B_{n, r}$ on the strings $w\left(k_{1}, \ldots, k_{n}\right)$. The property is then used to establish that the strings are pairwise inequivalent with respect to the language recognized by $B_{n, r}$.

Lemma 3 If $k_{i} \leq r$, then there exists a computation $C_{i}$ of the NFA $B_{n, r}$ which reaches the state $\left(i, k_{i}\right)$ at the end of the input $w\left(k_{1}, \ldots, k_{n}\right), 1 \leq i \leq n$. There is no computation of $B_{n, r}$ on $w\left(k_{1}, \ldots, k_{n}\right)$ that reaches a state $\left(i, k_{i}^{\prime}\right)$ with $k_{i}^{\prime}<$ 
$k_{i}$. Furthermore, if $k_{i}=r+1$, no computation of $B_{n, r}$ reaches at the end of $w\left(k_{1}, \ldots, k_{n}\right)$ a state where the first component is $i$.

Proof. We verify that a computation $C_{i}$ can reach state $\left(i, k_{i}\right), k_{i} \leq r$. First consider the case $i<n$. For $j=1, \ldots, i-1, a_{j}$ takes state $(j, 0)$ to $(j+1,0)$ and the next $k_{j}$ symbols $b_{j}$ are read using the self-loop in state $(j+1,0)$. In this way the computation reaches state $(i, 0)$ where we read $a_{i}$ using the self-loop and then reading the $k_{i}$ symbols $b_{i}$ the computation reaches $\left(i, k_{i}\right)$. In state $\left(i, k_{i}\right)$ the remaining suffix $a_{i+1} b_{i+1}^{k_{i+1}} \cdots a_{n-1} b_{n-1}^{k_{n-1}} b_{n}^{k_{n}}$ is consumed using the self-loops. Second, in the case $i=n$ similarly as above the computation after symbol $a_{n-1}$ reaches state $(n, 0)$, the symbols $b_{n-1}$ are read using self-loops and reading the $k_{n}$ symbols $b_{n}$ takes us to state $\left(n, k_{n}\right)$.

To verify the second part of the lemma we first observe the following. The only transitions of $B_{n, r}$ which move from a state $(i, j)$ to a state of the form $(i+1, j), 0 \leq j \leq r$, are on symbols $a_{i}$ and $c_{i}$. Note that since the distance $d_{r}$ associates cost $r+1$ to insertions and deletions, as well as to replacing $a_{i}$ or $c_{i}$ by any other symbol, the NFA $B_{n, r}$ does not have error transitions that change the first component of a state. Since $d_{r}\left(a_{i}, c_{i}\right)=0$, we can treat them as the same letter and for convenience, we refer only to $a_{i}$. Thus, the only way to reach a state $(q, j)$ for any $j \leq r$, is by taking transitions $\left((i, j), a_{i},(i+1, j)\right)$ on each occurrence of $a_{i}$ in $w\left(k_{1}, \ldots, k_{n}\right)$ for $i<q$. Otherwise, the computation remains in some state $\left(i^{\prime}, j\right)$ for $i^{\prime}<q$.

Now we show that there is no computation of $w\left(k_{1}, \ldots, k_{n}\right)$ that can reach a state $\left(j, k_{j}^{\prime}\right)$ with $k_{j}^{\prime}<k_{j}$. As discussed above, the only way for the computation to end in a state $(j, i), 0 \leq i \leq r$, is by reaching the state $(j, 0)$ when consuming the prefix $a_{1} b_{1}^{k_{1}} \cdots a_{j-1} b_{j-1}^{k_{j-1}}$ and then reading $a_{j}$ using a self-loop. There is no other way to reach a state $(j, i)$ for any $i$, since exiting the states with second components zero (corresponding to the original DFA) requires reading some $a_{j^{\prime}}$ with a self-loop, after which there is no transition which can be taken to move to a state $\left(j^{\prime}+1, i\right)$. If in the state $(j, 0)$ the symbol $a_{j}$ is not read with a self-loop then the first component becomes $j+1$ and we cannot reach a state $(j, i)$ with the remaining suffix. Thus, from $(j, 0)$ the NFA is forced to read the following $k_{j}$ symbols $b_{j}$ with error transitions, ending in the $k_{j}$-th level in the state $\left(j, k_{j}\right)$.

Exactly the same argument verifies that in the case $k_{j}=r+1$, no computation can end in a state where the first component is $j$. As above it is seen that to do this we must in the state $(j, 0)$ read the symbol $a_{j}$ with a self-loop and after attempting to read the following $r+1$ symbols $b_{j}$ with an error transition the computation becomes undefined.

With the previous lemma we can now establish a lower bound for the state complexity of the neighbourhoods of $L\left(A_{n}\right)$ with respect to the quasi-distance $d_{r}$.

Lemma 4 Let $A_{n}$ be the DFA as in Figure 1. The strings $w\left(k_{1}, \ldots, k_{n}\right), 0 \leq$ $k_{i} \leq r+1,1 \leq i \leq n$, are all pairwise inequivalent with respect to the Kleene congruence of $E\left(L\left(A_{n}\right), d_{r}, r\right)$. 
Proof. We consider two distinct strings $w\left(k_{1}, \ldots, k_{n}\right)$ and $w\left(k_{1}^{\prime}, \ldots, k_{n}^{\prime}\right)$ with $0 \leq k_{i}, k_{i}^{\prime} \leq r+1$ for $i=1, \ldots, n$. There exists an index $j$ such that $k_{j} \neq k_{j}^{\prime}$ and without loss of generality, we have $k_{j}<k_{j}^{\prime}$. To distinguish the strings $w\left(k_{1}, \ldots, k_{n}\right)$ and $w\left(k_{1}^{\prime}, \ldots, k_{n}^{\prime}\right)$ consider the word $z=b_{j}^{r-k_{j}} a_{j} \cdots a_{n-1}$. The string $z$ is well-defined since $k_{j}<k_{j}^{\prime} \leq r+1$ and so $r-k_{j} \geq 0$.

Let $B_{n, r}$ be the NFA constructed for $E\left(L(A), d_{r}, r\right)$ as in (2). We claim that $w\left(k_{1}, \ldots, k_{n}\right) \cdot z \in L\left(B_{n, r}\right)$ but $w\left(k_{1}^{\prime}, \ldots, k_{n}^{\prime}\right) \cdot z \notin L\left(B_{n, r}\right)$. We note that by Lemma $3, B_{n, r}$ has a computation on $w\left(k_{1}, \ldots, k_{n}\right)$ that ends in state $\left(j, k_{j}\right)$. Note that $k_{j} \leq r$. When continuing the computation on the string $z$, by reading the $r-k_{j}$ symbols $b_{j}$ 's, the machine is taken to the state $(j, r)$. Then, reading the suffix $a_{j} \cdots a_{n-1}$ takes the machine to the accepting state $(n, r)$.

To show $w\left(k_{1}^{\prime}, \ldots, k_{n}^{\prime}\right) \cdot z \notin L\left(B_{n, r}\right)$, we consider from which states of $B_{n, r}$ an accepting state, that is, a state with first component $n$ is reachable on the string $z$. We recall that in $B_{n, r}$ the transitions on $b_{j}$ cannot change the first component of the state. (According to the definition of $B_{n, r}$ the reason for this is that $d_{r}$ associates cost $r+1$ to insertion/deletion or to subsitute a symbol $a_{i}$, $c_{i}$ by $b_{j}$.)

Thus, for $B_{n, r}$ to reach an accepting state (with first component $n$ ) on the string $w\left(k_{1}^{\prime}, \ldots, k_{n}^{\prime}\right) \cdot z$, a computation must reach a state of the form $\left(j, \ell_{j}\right)$ on the prefix $w\left(k_{1}^{\prime}, \ldots, k_{n}^{\prime}\right)$. By Lemma 3 , this is possible only if $\ell_{j} \geq k_{j}^{\prime}$. From state $\left(j, \ell_{j}\right), \ell_{j} \geq k_{j}^{\prime}$, reading the substring $b_{j}^{r-k_{j}}$ takes the machine to an undefined state, as it is not possible to make $r-k_{j}$ error transitions on $b_{j}$ in a state where the second component is $\ell_{j}>k_{j}$. This means that $B_{n, r}$ cannot accept the string $w\left(k_{1}^{\prime}, \ldots, k_{n}^{\prime}\right) \cdot z$.

Thus, each string $w\left(k_{1}, \ldots, k_{n}\right), 0 \leq i \leq r+1,1 \leq i \leq n$, defines a distinct equivalence class of $\equiv_{E\left(L(A), d_{r}, r\right)}$.

As a corollary of the proof of Lemma 4 we get also a lower bound for the state complexity of the neighbourhood of an NFA-language with respect to an additive distance.

Lemma 5 For $n, r \in \mathbb{N}$ there exists an additive distance $d_{r}^{\prime}$ and an NFA $A_{n}^{\prime}$ over an alphabet $\Sigma_{n}^{\prime}$ of size $2 n-1$ such that

$$
\operatorname{sc}\left(E\left(L\left(A_{n}^{\prime}\right), d_{r}^{\prime}, r\right)\right) \geq(r+2)^{n}-1 .
$$

Proof. Choose $\Sigma_{n}^{\prime}=\left\{a_{1}, \ldots, a_{n-1}, b_{1}, \ldots, b_{n}\right\}$ and $d_{r}^{\prime}$ is the restriction of $d_{r}$ to the alphabet $\Sigma_{n}^{\prime}$ (where $d_{r}$ is the quasi-distance of Lemma 2). The function $d_{r}^{\prime}$ does not assign distance zero to any pair of distinct elements.

The NFA $A_{n}^{\prime}$ is obtained from the DFA $A_{n}$ in Figure 1 by replacing all $c_{i}$ transitions by $a_{i}$-transitions, $1 \leq i \leq n-1$. Thus, $A_{n}^{\prime}$ is nondeterministic. An NFA $B_{n, r}^{\prime}$ for the neighbourhood $E\left(L\left(A_{n}^{\prime}\right), d_{r}^{\prime}, r\right)$ is obtained from the NFA $B_{n, r}$ in (2) simply by omitting all transitions on $c_{i}, 1 \leq i \leq n-1$. Note that in $B_{n, r}$ the transitions on $c_{i}$ exactly coincide with the transitions on $a_{i}, 1 \leq i \leq n-1$, reflecting the situation that $d_{r}\left(a_{i}, c_{i}\right)=0$.

The strings $w\left(k_{1}, \ldots, k_{n}\right)$ (as in (3)) did not involve any symbols $c_{i}$, and the proof of Lemma 4 remains the same, word for word, just by replacing $B_{n, r}$ 
with $B_{n, r}^{\prime}$. There are in total $(r+2)^{n}$ strings $w\left(k_{1}, \ldots, k_{n}\right), 0 \leq k_{i} \leq r+1$, $i=1, \ldots, n$, and the string $w(r+1, \ldots, r+1)$ corresponds to the dead state which can be omitted.

Now putting together Lemma 4, Lemma 5, and Proposition 2, we have:

Theorem 1 If $d$ is an additive quasi-distance, $A$ is an NFA with $n$ states and $r \in \mathbb{N}$, then

$$
\operatorname{sc}\left(E(L(A), d, r) \leq(r+2)^{n}-1 .\right.
$$

There exists an additive quasi-distance $d_{r}$ and a DFA $A$ with $n$ states over an alphabet of size $3 n-2$ such that $\operatorname{sc}\left(E\left(L(A), d_{r}, r\right)=(r+2)^{n}-1\right.$.

There exists an additive distance $d_{r}^{\prime}$ and an NFA $A^{\prime}$ with $n$ states over an alphabet of size $2 n-1$ such that $\operatorname{sc}\left(E\left(L\left(A^{\prime}\right), d_{r}^{\prime}, r\right)=(r+2)^{n}-1\right.$.

The lower bound construction has the trade-off of either using a DFA and a quasi-distance or an NFA and a distance, respectively. It would be interesting to know whether or not the general upper bound can be improved for neighbourhoods of an $n$ state DFA language defined by an additive distance.

\section{State Complexity of Approximate Pattern Match- ing}

We consider an extension of the pattern matching problem with mismatches in the sense of El-Mabrouk [7]. For a given finite automaton $A$ and an additive quasi-distance $d$ we construct a DFA for the language $\Sigma^{*} E(L(A), d, r) \Sigma^{*}$, that is, the set of strings that contain a substring within distance $r$ from a string of $L(A)$. The construction gives an upper bound for the pattern matching problem and using a modification of the constructions in the previous section we show that the upper bound is optimal.

Lemma 6 Let $A=\left(Q, \Sigma, \delta, q_{0}, F_{A}\right)$ be an n-state $N F A$ with $k \geq 1$ final states and $d$ be an additive quasi-distance. Then the language

$$
L_{1}=\Sigma^{*} E(L(A), d, r) \Sigma^{*}
$$

can be recognized by a DFA $B$ with $(r+2)^{n-1-k}+1$ states.

Proof. Let $Q=\left\{q_{0}, q_{1}, \ldots, q_{n-1}\right\}$. If $q_{0} \in F_{A}$, then $L_{1}=\Sigma^{*}$ and there is nothing to prove. Thus, in the following we can assume that $F=\left\{q_{n-k}, q_{n-k+1}, \ldots, q_{n-1}\right\}$, $1 \leq k \leq n-1$. Furthermore, without loss of generality we assume that

$$
\left(\forall w \in \Sigma^{*}\right) \delta\left(q_{0}, w\right) \cap F_{A} \neq \emptyset \text { implies } d(\varepsilon, w)>r .
$$

If the above condition does not hold, $\varepsilon \in E(L(A), d, r)$ and there is nothing to prove.

Define $B=\left(P, \Sigma, \gamma, p_{0}, F_{B}\right)$ with set of states

$$
P=\left\{\left(i_{1}, \ldots, i_{n-k-1}\right) \mid 0 \leq i_{j} \leq r+1, j=1, \ldots, n-k-1\right\} \cup\left\{p_{f}\right\},
$$


the initial state is $p_{0}=\left(h_{1}, \ldots, h_{n-k+1}\right)$ where

$$
h_{z}=\inf \left\{d(\varepsilon, w) \mid q_{z} \in \delta\left(q_{0}, w\right)\right\}, \quad 1 \leq z \leq n-k-1,
$$

and the set of final states is defined as $F_{B}=\left\{p_{f}\right\}$. Note that by (4) we know that $\varepsilon \notin L_{1}$. Next we define the transitions of $B$. First, $\gamma\left(p_{f}, b\right)=p_{f}$ for all $b \in \Sigma$. For $\mathbf{p}=\left(i_{1}, \ldots, i_{n-k-1}\right) \in P, 0 \leq i_{z} \leq r+1, z=1, \ldots, n-k-1$, and $b \in \Sigma$ we define

(i) $\gamma(\mathbf{p}, b)=p_{f}$ if $(\exists 1 \leq z \leq n-k-1)\left(\exists w \in \Sigma^{*}\right) \delta\left(q_{z}, w\right) \cap F_{A} \neq \emptyset$ and $i_{z}+$ $d(b, w) \leq r$

(ii) and if the conditions in (i) do not hold, then $\gamma(\mathbf{p}, b)=\left(j_{1}, \ldots, j_{n-k-1}\right)$, where, for $x=1, \ldots, n-k-1$,

$$
\begin{aligned}
j_{x}= & \inf \left[\left\{i_{z}+d(b, w) \mid q_{x} \in \delta\left(q_{z}, w\right), 1 \leq z \leq n-k-1\right\}\right. \\
& \left.\cup\left\{d(b, w) \mid q_{x} \in \delta\left(q_{0}, w\right)\right\}\right] .
\end{aligned}
$$

In a state of the form $\left(i_{1}, \ldots, i_{n-k-1}\right) \in P$, the component $i_{z}, 1 \leq z \leq$ $n-k-1$, keeps track of the smallest distance $d\left(u_{\text {suf }}, w\right)$ where $u_{\text {suf }}$ is a suffix of the input processed up to that point and $w$ is a string that in $A$ takes the initial state $q_{0}$ to state $q_{z}$. The smallest error between the suffix $\varepsilon$ and a string that in $A$ reaches $q_{0}$ is always zero and this value is not stored in the state of $B$. If the computation has found a substring in $E(L(A), d, r)$, the state of $B$ will be $p_{f}$.

Roughly speaking, $B$ is looking for a substring of the input that belongs to $E(L(A), d, r)$. For this purpose, for all non-final states $q_{z}$ of $A$, the deterministic computation of $B$ keeps track of the smallest cumulative error between a string that takes $q_{0}$ to $q_{z}$ and any suffix of the input processed thus far. Note that for the initial state $q_{0}$ this value is always zero and, hence, the states of $P$ store the cumulative error only for the nonfinal states $q_{1}, \ldots, q_{n-k-1}$. When $B$ has found a substring belonging to $E(L(A), d, r)$ the computation goes to the final state $p_{f}$ and after that accepts an arbitrary suffix.

We show that in computations of $B$ in a state of the form $\left(i_{1}, \ldots, i_{n-k-1}\right) \in$ $P$, the component $i_{z}, 1 \leq z \leq n-k-1$, is the smallest distance $d\left(u_{\text {suf }}, w\right)$ between a suffix of the input processed so far and a string $w$ that takes the initial state $q_{0}$ of $A$ to the state $q_{z}$.

The initial state $p_{0}$ is chosen so that the above condition is satisfied at the beginning of the computation. Note that, by (4), we can assume that $\varepsilon \notin E(L(A), d, r)$. Now consider a string $u b, u \in \Sigma^{*}, b \in \Sigma$, and inductively assume that the state of $B$ satisfies the claimed condition after processing the prefix $u$.

If $B$ has reached the final state $p_{f}$ after processing $u$, the rest of the computation remains in state $p_{f}$ and we are done. Assume then that the state of $B$ after processing $u$ is $\left(i_{1}, \ldots, i_{n-k-1}\right)$. By the inductive assumption, $i_{z}$ is the smallest cumulative error between a suffix of $u$ and any string that in $A$ takes the initial state to $q_{z}, 1 \leq z \leq n-k-1$. This means that, according to (i), the transition on $b$ goes to the final state $p_{f}$ if the smallest cumulative error between 
a suffix of $u b$ and a string of $L(A)$ is at most $r$. Note that, again by (4), we know that the suffix cannot be $\varepsilon$.

On the other hand, if a string satisfying these conditions does not exist, the rule (ii) changes the tuple $\left(i_{1}, \ldots, i_{n-k-1}\right)$ to a tuple $\left(j_{1}, \ldots, j_{n-k-1}\right)$ where $j_{x}$ is the smallest value of $i_{z}+d(b, w)$ where the string $w$ takes in the NFA $A$ the state $q_{z}$ to $q_{x}, 0 \leq z \leq n-k-1$. Corresponding to $q_{0}$ the value $i_{0}$ is zero because the pattern in $E(L(A), d, r)$ to be matched can begin at any position. Thus the rule (ii) updates the tuple of values correctly to record the smallest cumulative error between a suffix of $u b$ and a string that in $A$ takes the initial state, respectively, to $q_{1}, \ldots, q_{n-k-1}$.

By modifying the construction used in the proof of Lemma 5 (and Lemma 4) we give a lower bound that matches the upper bound from Lemma 6 .

Lemma 7 For $n, r \in \mathbb{N}$, there exist an additive distance $d$ and an NFA $A$ with $n$ states defined over an alphabet $\Sigma$ of size $2 n-1$ such that the minimal DFA for $\Sigma^{*} E(L(A), d, r) \Sigma^{*}$ must have at least $(r+2)^{n-2}+1$ states.

Proof. Choose $\Sigma_{n}=\left\{a_{1}, \ldots, a_{n-1}, b_{1}, \ldots, b_{n}\right\}$ and let $A_{n}^{\prime}$ and $d_{r}^{\prime}$ be as in the proof of Lemma 5. Let $B_{n, r}^{\prime}$ be the NFA constructed for $E\left(L\left(A_{n}^{\prime}\right), d_{r}^{\prime}, r\right)$ in the proof of Lemma $5 ; B_{n, r}^{\prime}$ is obtained from the NFA of Fig. 2 by omitting all the transitions on $c_{i}$ 's. For $0 \leq k_{i} \leq r+1, i=1,2, \ldots, n-2$, define

$$
u\left(k_{1}, k_{2}, \ldots, k_{n-2}\right)=a_{1} b_{1}^{k_{1}} a_{2} b_{2}^{k_{2}} \cdots a_{n-2} b_{n-2}^{k_{n-2}} .
$$

Using the notations of (3) we have $u\left(k_{1}, \ldots, k_{n-2}\right) \cdot a_{n-1}=w\left(k_{1}, k_{2}, \ldots, k_{n-2}, 0,0\right)$.

We claim that the strings $u\left(k_{1}, \ldots, k_{n-2}\right)$ are all pairwise inequivalent with respect to the Kleene congruence of $\Sigma_{n}^{*} E\left(L\left(A_{n}^{\prime}\right), d_{r}^{\prime}, r\right) \Sigma_{n}^{*}$. Consider two strings $u\left(k_{1}, \ldots, k_{n-2}\right)$ and $u\left(k_{1}^{\prime}, \ldots, k_{n-2}^{\prime}\right)$ where for some $1 \leq j \leq n-2, k_{j}<k_{j}^{\prime}$.

Choose $z=b_{j}^{r-k_{j}} a_{j+1} \cdots a_{n-1}$. As in the proof of Lemma 3 it is observed that $B_{n, r}^{\prime}$ has a computation on $u\left(k_{1}, \ldots, k_{n-2}\right)$ that reaches state $\left(j, k_{j}\right)$, and a computation started from state $\left(j, k_{j}\right)$ on input $z$ can reach the accepting state $(n, r)$. Thus, $u\left(k_{1}, \ldots, k_{n-2}\right) \cdot z \in L\left(B_{n, r}^{\prime}\right)=E\left(L\left(A_{n}^{\prime}\right), d_{r}^{\prime}, r\right)$. We claim that

$$
u\left(k_{1}^{\prime}, \ldots, k_{n-2}^{\prime}\right) \cdot z \notin \Sigma_{n}^{*} E\left(L\left(A_{n}^{\prime}\right), d_{r}^{\prime}, r\right) \Sigma_{n}^{*} .
$$

Note that the string $u\left(k_{1}^{\prime}, \ldots, k_{n-2}^{\prime}\right) \cdot z$ contains exactly one occurrence of both $a_{1}$ and $a_{n-1}$ and these are, respectively, the first and the last symbol of the string. Since the distance $d_{r}^{\prime}$ associates cost $r+1$ to any operation that substitutes, deletes or inserts a symbol $a_{i}$, if the negation of (5) holds then the only possibility is that $u\left(k_{1}^{\prime}, \ldots, k_{n-2}^{\prime}\right) \cdot z$ must be in $E\left(L\left(A_{n}^{\prime}\right), d_{r}^{\prime}, r\right)$. This, in turn, is possible only if the computation of $B_{n, r}^{\prime}$ on the prefix $u\left(k_{1}^{\prime}, \ldots, k_{n-2}^{\prime}\right)$ ends in a state of the form $(j, x), 0 \leq x \leq r$. Now Lemma 3 implies that the second component $x$ must be at least $k_{j}^{\prime}$ and it follows that the computation on the suffix $z$ cannot end in an accepting state. (Lemma 3 uses $B_{n, r}$ but the same argument applies here because $B_{n, r}$ equals $B_{n, r}^{\prime}$ when we omit the $c_{i}$-transitions.)

Finally we note that none of the strings $u\left(k_{1}, \ldots, k_{n-2}\right), 0 \leq k_{i} \leq r+1$, is in $\Sigma_{n}^{*} E\left(L\left(A_{n}^{\prime}\right), d_{r}^{\prime}, r\right) \Sigma_{n}^{*}$ and hence they are not equivalent with $a_{1} a_{2} \cdots a_{n-1}$ which then gives the one additional equivalence class. 
Combining the previous lemmas we can state the main result of this section.

Theorem 2 Let $d$ be an additive quasi-distance on $\Sigma^{*}$. For any $n$-state NFA $A$ and $r \in \mathbb{N}$ we have

$$
\operatorname{sc}\left(\Sigma^{*} \cdot E(L(A), d, r) \cdot \Sigma^{*}\right) \leq(r+2)^{n-2}+1 .
$$

For given $n, r \in \mathbb{N}$ there exist an additive distance $d_{r}$ and an n-state NFA $A$ defined over an alphabet of size $2 n-1$ such that $\operatorname{sc}\left(\Sigma^{*} E\left(L(A), d_{r}, r\right)\right) \Sigma^{*}=$ $(r+2)^{n-2}+1$.

Proof. The upper bound of Lemma 6 is maximized by an NFA with one final state as $(r+2)^{n-2}+1$. The lower bound follows by Lemma 7 .

Finally we can note that similarly as in Theorem 1, in the lower bound result of Lemma 7 we could select $A$ to be a DFA if we allow $d_{r}$ to be a quasi-distance.

Corollary 1 For given $n, r \in \mathbb{N}$ there exist an additive quasi-distance $d_{r}^{\prime}$ and an $n$-state DFA A defined over an alphabet of size $3 n-2$ such that $\operatorname{sc}\left(\Sigma^{*} E\left(L(A), d_{r}^{\prime}, r\right)\right) \Sigma^{*}=$ $(r+2)^{n-2}+1$.

Recall that Brzozowski et al. [4] have shown that, for an $n$-state DFA language $L$, the worst case state complexity of the two-sided ideal $\Sigma^{*} L \Sigma^{*}$ is $2^{n-2}+1$. This corresponds to the case of having error radius zero $(r=0)$ in Corollary 1. Differing from here, Brzozowski et al. [4] have defined state complexity (which they call quotient complexity) in terms of the size of the minimal complete DFA. However, the lower bound with $r=0$ coincides with the result of Corollary 1 because, for a nonempty language $L$, the minimal DFA for $\Sigma^{*} L \Sigma^{*}$ does not have a dead state. Lemma 7 and Corollary 1 require a linear size alphabet whereas the tight lower bound for the error free case is obtained with a three letter alphabet [4].

\section{Conclusion}

We have given a tight lower bound construction for the state complexity of a neighbourhood of a regular language. The construction uses a variable alphabet of size linear in the number of states of the NFA. The main open problem for further work is to develop lower bounds for neighbourhoods of languages over a fixed alphabet. For radius one Hamming neighbourhoods an improved upper bound and a good lower bound using a binary alphabet were given by Povarov [18].

Our lower bound for the approximate pattern matching problem was obtained by modifying the lower bound construction for neighbourhoods of a regular language. This was, roughly speaking, made possible by the choice of the distance function and the language definition where the strings must contain the symbols $a_{1}, \ldots, a_{n-1}$ in this particular order. Similar constructions will be more challenging if restricted to a fixed alphabet. 


\section{References}

[1] Benedikt, M., Puppis, G., Riveros, C.: Bounded repairability of word languages. J. Comput. System Sciences 79 (2013) 1302-1321

[2] Bordihn, H., Holzer, M., Kutrib, M.: Determination of finite automata accepting subregular languages. Theoretical Computer Science 410 (2009) 3209-3249

[3] Boyer, R.S., Moore, J.S.: A fast string searching algorithm. Communications of ACM 20 (1977) 762-772

[4] Brzozowski, J., Jirásková, G., Li, B.: Quotient Complexity of Ideal Languages. In: Latin American Theoretical Informatics Symposium. (2010) $208-221$

[5] Calude, C.S., Salomaa, K., Yu, S.: Additive Distances and Quasi-Distances Between Words. Journal of Universal Computer Science 8(2) (2002) 141152

[6] Deza, M.M., Deza, E.: Encyclopedia of Distances. Springer-Verlag, BerlinHeidelberg (2009)

[7] El-Mabrouk, N.: On the size of minimal automata for approximate string matching. Technical report, Institut Gaspard Monge, Université de Marne la Vallée, Paris (1997)

[8] Han, Y.-S., Ko, S.-K., Salomaa, K.: The edit distance between a regular language and a context-free language. International Journal of Foundations of Computer Science 24 (2013) 1067-1082

[9] Kari, L., Konstantinidis, S.: Descriptional complexity of error/edit systems. Journal of Automata, Languages, and Combinatorics 9 (2004) 293309

[10] Kari, L., Konstantinidis, S., Kopecki, S., Yang, M.: An efficient algorithm for computing the edit distance of a regular language via input-altering transducers. CoRR abs/1406.1041 (2014)

[11] Konstantinidis, S.: Transducers and the properties of error detection, errorcorrection, and finite-delay decodability. Journal of Universal Computer Science 8 (2002) 278-291

[12] Konstantinidis, S.: Computing the edit distance of a regular language. Information and Computation 205 (2007) 1307-1316

[13] Konstantinidis, S., Silva, P.: Maximal error-detecting capabilities of formal languages. J. Automata, Languages, and Combinatorics 13 (2008) 55-71 
[14] Konstantinidis, S., Silva, P.: Computing maximal error-detecting capabilities and distances of regular languages. Fundamenta Informaticae 101 (2010) 257-270

[15] Levenshtein, V.I.: Binary codes capable of correcting deletions, insertions, and reversals. Soviet Physics Doklady 10(8) (1966) 707-710

[16] Ng, T., Rappaport, D., Salomaa, K.: Quasi-Distances and Weighted Finite Automata. In: Descriptional Complexity of Formal Systems, DCFS'15, Waterloo, Ontario, June 25-27, 2015, Lect. Notes Comput. Sci. 9118 (2015) 209-219

[17] Pighizzini, G.: How hard is computing the edit distance? Information and Computation 165 (2001) 1-13

[18] Povarov, G.: Descriptive Complexity of the Hamming Neighborhood of a Regular Language. In: Language and Automata Theory and Applications. (2007) 509-520

[19] Salomaa, K., Schofield, P.: State Complexity of Additive Weighted Finite Automata. International Journal of Foundations of Computer Science 18(06) (December 2007) 1407-1416

[20] Schulz, K.U., Mihov, S.: Fast string correction with Levenshtein automata. International Journal on Document Analysis and Recognition 5 (2002) 6785

[21] Shallit, J.: A Second Course in Formal Languages and Automata Theory, Cambridge University Press (2009)

[22] Shamkin, S.: Descriptional complexity of Hamming neighbourhoods of finite languages (in Russian). M.Sc. thesis, Ural Federal University, Ekaterinburg, Russia (2011)

[23] Yu, S.: Regular languages, in: Handbook of Formal Languages, Vol. I, (G. Rozenberg, A. Salomaa, Eds.), Springer, 1997, pp. 41-110 